\title{
Regenerative medicine for the treatment of musculoskeletal overuse injuries in competition horses
}

\author{
Paola Torricelli • Milena Fini • Giuseppe Filardo • \\ Matilde Tschon • Mario Pischedda • \\ Alessandra Pacorini $\cdot$ Elizaveta Kon • Roberto Giardino
}

Received: 30 December 2010 / Accepted: 17 February 2011 /Published online: 11 March 2011

(C) Springer-Verlag 2011

\begin{abstract}
Purpose Tissue repair in musculoskeletal injuries is often a slow and sometimes incomplete process. Regenerative medicine based on the use of growth factors (GFs) and cell therapy is aimed at improving the quality and speed of tendon and ligament healing. The aim of this study was to evaluate the potential for the administration of a combination of autologous platelet-rich plasma (PRP) and freshly isolated bone marrow mononucleated cells (BMMNCs) in 13 competition horses affected by overuse musculoskeletal injuries (suspensory ligament desmopathy and superficial flexor tendinopathy) and refractory to other therapies.

Methods After ultrasonographic localisation of the lesion, the autologous BMMNC suspension and PRP were injected directly into the core lesion. BMMNC and platelet count as well as growth factors in PRP were measured to determine factors influencing the clinical outcome.

Results Horses showed a marked improvement in their degree of lameness and $84.6 \%$ were able to return to competition. Among all the factors studied, the platelet concentration
\end{abstract}

P. Torricelli $\cdot$ M. Fini $\cdot$ M. Tschon $\cdot$ R. Giardino

Laboratory of Preclinical and Surgical Studies,

Research Institute Codivilla-Putti, Rizzoli Orthopaedic Institute,

Via di Barbiano 1/10,

40136 Bologna, Italy

G. Filardo $\cdot$ E. Kon $(\bowtie)$

Laboratory of Biomechanics and Technology Innovation- III

Clinic, Rizzoli Orthopaedic Institute,

Via di Barbiano 1/10,

40136 Bologna, Italy

e-mail: e.kon@biomec.ior.it

M. Pischedda $\cdot$ A. Pacorini

Veterinary Studio,

via Marzabotto 12, Rastignano,

Bologna, Italy predicted the healing time: significantly faster recovery ( $p=$ 0.049) was observed in cases of PRP with more than $750 \times$ $10^{3} / \mu$ l platelets.

Conclusions Competition horses are involved in highly demanding activities, thus being a similar model for the high mechanical overload typical of human athletes. The promising results obtained suggest that this combined biological approach may be useful even for the treatment of recalcitrant overuse musculoskeletal injuries in highly demanding patients if the appropriate dose of cells and GFs is applied.

\section{Introduction}

Chronic painful musculoskeletal disorders are common debilitating conditions, and the real cost of these injuries to society is remarkable [1]. In sports patients or professional athletes the impact on life and work is even greater, and the fast recovery of full efficiency and return to competition is of primary importance. Tissue repair in musculoskeletal injuries is often a slow and sometimes incomplete process, and the treatment options available may offer incomplete recovery and difficulties in returning to high activity level $[2,3]$.

Recently, regenerative medicine and tissue engineering have focused on the use of growth factors (GFs) and cell-based therapy to improve the quality and speed of healing in tendons and ligaments [4]. GFs are important signalling molecules involved in connective tissue healing, regulating many aspects of cellular metabolism, such as cell proliferation and differentiation, and extracellular matrix synthesis [5]. Platelet products, such as platelet-rich plasma (PRP), are a great reservoir of GFs and cytokines. In fact, activated platelets secrete GFs, such as platelet-derived growth factor (PDGF), transforming growth factors (TGFs), insulinlike growth factor (IGF), epidermal growth factor (EGF), 
vascular endothelial growth factor (VEGF), and many other bioactive molecules that enhance angiogenesis and increase the proliferation of undifferentiated mesenchymal stem cells (MSCs) [6, 7].

MSCs are known to be pluripotent precursors of connective tissues, and they may play an important role in favouring musculoskeletal tissue healing [8, 9]. The delivery of bone marrow mononucleated cells (BMMNCs) has been described as an alternative procedure to grafting MSCs in injured tissues, and preclinical studies have shown promising results for the treatment of tendinopathy $[10,11]$. Analogous animal models have also suggested the usefulness of platelet concentrate for the treatment of tendon damage, leading to the application of PRP in humans, where the first reports seem to confirm the potential of PRP to increase the tissue healing potential $[12,13]$. However, both treatment approaches raise controversies, due to the lack of scientifically robust studies demonstrating their effectiveness unequivocally, contradictory results in some therapeutic applications, and to the only partial clinical efficacy of these biological regenerative methods documented in other studies [4, 14].

A combination of both autologous MSCs and platelet derived GFs, locally administrated, could be a valid solution in order to further increase the potential of these techniques, enhancing synergically the regenerative process as suggested by some recent studies $[15,16]$.

The aim of our study was to evaluate the potential of the combined biological procedure of autologous PRP and freshly isolated BMMNCs administration in competition horses affected by overuse musculoskeletal injuries and refractory to other therapies. A secondary aim was to determine factors influencing the clinical outcome, in order to give indications for future studies and improve the potential of this regenerative approach.

\section{Materials and methods}

Study design

Thirteen adult competition horses (Table 1) were enrolled in the study with the informed consent of their owners. The pathologies arose between two and 12 months before BMMNCs and PRP treatment. The horses had been previously treated with rest, pharmacological (steroidal and non steroidal anti-inflammatory drugs, blistering, hyaluronic acid injections) and non-pharmacological (rehabilitation protocols) therapies without improvement.

Horses were examined clinically (lameness grade, standing and moving anamnesis, presence of pain, oedema or swelling) and by an ultrasound system (Aloka $500 \mathrm{SSD}$, Aloka Holding Europe, Zug, Switzerland) by the same veterinarian, including perineural and intra-articular regional anaesthesia when necessary to precisely localise the lesion. The lameness grade score ranged from 0 to 5 in accordance with the American Association for Equine Practitioner's criteria (1991). Horses were treated with their own PRP and one-step isolated BMMNCs. After a 12-month follow-up, lameness grade and ultrasonography were re-evaluated. Adverse events and healing time, that is the measure of time required to return to pre-injury activity level, other than return to competition, were also recorded.

\section{PRP preparation}

Equine whole venous blood was collected with sodiumcitrate. PRP was obtained by two sequential centrifugations (5 min at $200 \mathrm{~g}$ and $15 \mathrm{~min}$ at $1000 \mathrm{~g}$ ). For activation a sterile $10 \%$ calcium chloride solution $\left(\mathrm{CaCl}_{2}\right.$, SigmaAldrich, St Louis, MO, USA) was added to PRP immediately before transplanting to initiate clot formation and to
Table 1 Codes, sex, age, diagnosis and clinical evaluations of the 13 competition horses treated

\begin{tabular}{llllll}
\hline Horse & Sex & Age (y) & Diagnosis & Lameness grade & Clinical signs \\
\hline H1 & Male & 15 & Suspensory ligament desmopathy & $4 / 5$ & Oedema, swelling, pain \\
H2 & Female & 13 & Suspensory ligament desmopathy & $2 / 5$ & None \\
H3 & Male & 16 & Suspensory ligament desmopathy & $3 / 5$ & Oedema, swelling, pain \\
H4 & Female & 9 & Superficial flexor tendinopathy & $2 / 5$ & Oedema, swelling, pain \\
H5 & Female & 6 & Superficial flexor tendinopathy & $1 / 5$ & Swelling \\
H6 & Female & 5 & Superficial flexor tendinopathy & $2 / 5$ & Swelling \\
H7 & Female & 8 & Suspensory ligament desmopathy & $2 / 5$ & None \\
H8 & Male & 12 & Suspensory ligament desmopathy & $2 / 5$ & None \\
H9 & Male & 7 & Suspensory ligament desmopathy & $1 / 5$ & Swelling \\
H10 & Female & 10 & Suspensory ligament desmopathy & $2 / 5$ & None \\
H11 & Female & 8 & Suspensory ligament desmopathy & $1 / 5$ & Swelling \\
H12 & Male & 12 & Suspensory ligament desmopathy & $2 / 5$ & Swelling \\
H13 & Male & 16 & Superficial flexor tendinopathy & $2 / 5$ & Swelling \\
\hline
\end{tabular}


form a three-dimensional fibrin gel able to retain BMMNCs into the lesion site. Platelets were counted on whole blood and PRP. The platelet concentration factor was calculated by dividing the number of platelets in the PRP and the number of platelets in the whole blood.

Growth factor measurements

Aliquots of basal plasma and $\mathrm{CaCl}_{2}$-activated PRP (aPRP) were assayed in eight horses within one hour from activation to measure the GF content by means of specific enzyme-linked immunoassay (Quantikine Immunoassay, R\&D Systems, MN, USA) for transforming growth factor- $\beta 1$ (TGF- $\beta 1$ ), platelet derived growth factor-AB (PDGF-AB), epidermal growth factor (EGF), vascular endothelial growth factor (VEGF), insulin-like growth factor (IGF) and interleukin $1 \beta$ (IL-1 $\beta$ ) following manufacturer's instruction. Cross reaction of antibodies of these kits with equine GFs has been reported [17].

\section{BMMNCs isolation}

A sterile 50-ml syringe containing heparin was attached to a 16-gauge bone-marrow needle inserted into the posterior tuber coxae: bone marrow $(40 \mathrm{ml})$ was aspirated and immediately processed by isopyknic centrifugation. Briefly, cells were isolated by density gradient medium (FicollPaque, density 1.077 g per ml, Sigma-Aldrich, MO, USA), washed, counted in a haemocytometric chamber and resuspended in PRP for immediate transplant.

\section{PRP-BMMNC transplant and post-treatment protocol}

The affected area of the limb of each horse was shaved and disinfected (Betadine, Meda, Milan, Italy). The horses were locally sedated (Lidocaine 2\%, Aziende Chimiche Angelini, Rome, Italy). After ultrasonographic localisation of the lesion site, the autologous BMMNCs suspension and aPRP (range, 4-7 ml) were slowly injected directly into the core lesion.

The animals were clinically evaluated 48 hours after the injection and then weekly. All horses underwent a one-year follow-up evaluation. The animals were maintained at a very low-level of exercise activity (hand walking) for a one-month period. Then, when symptom-free, after a warm-up walk, they trotted for five minutes, increasing intensity every week until entering a complete training protocol including walking, trotting and galloping.

Statistical analysis

All continuous variables were expressed in terms of mean \pm standard deviation of the mean if normally distributed, median and 25 th and 75 th percentiles were used otherwise. The
Kolmogorov Smirnov test was performed to test the hypotheses about normality. The Wilcoxon test (exact method) for small samples was performed to test the differences of means between basal level and follow-up. Mann Whitney test (exact method) was used to test the differences between groups. Spearman rank correlation analysis was performed to investigate relationships between two quantitative measurements. For all tests $p<0.05$ was considered significant. Statistical analysis was performed by means of SPSS 15.0.

\section{Results}

\section{PRP and BMMNCs}

Data on PRP preparation and BMMNCs isolation for each animal are summarised in Table 2. The mean number of platelets was $144 \times 10^{3} / \mu$ l in the whole blood and $751 \times 10^{3} / \mu 1$ after centrifugation (concentration range, 3.5-7.3, mean 5.4). The number of BMMNCs differed greatly, varying between 0.8 and $4 \times 10^{6}$ cells, mean $1.9 \times 10^{6}$.

\section{Growth factor measurements}

Evaluation of GFs and IL-1ß in plasma and aPRP samples was performed in eight horses and is summarised in Table 3. The analysis of results revealed that when PRP was activated, the platelet degranulation was responsible for the release of a greater amount of GFs in the supernatant as reflected by the higher values of GFs achieved in the aPRP samples in comparison with plasma values $(p<0.0005)$. IL-1ß values also significantly increased in aPRP in comparison with basal plasma.

\section{Clinical outcome}

None of the enrolled animals had any major adverse reactions as a result of the transplant, either locally or systemically. Two of them developed swelling after the treatment that gradually resolved within one month. The rehabilitation program was well tolerated.

All horses showed a marked improvement $(p<0.0005)$ in their degree of lameness: from a mean of 2.0 (range, 1-4) to a complete absence of symptoms at 12 months. All animals treated returned to pre-injury activity level after 2-12 months of rehabilitation. Eleven of them $(84.6 \%)$ were also able to return to competition, whereas two of them (the ones who experienced adverse events) did not go back to races (Fig. 1). Ultrasonographic examination was negative in all animals (Fig. 2).

Further analysis was performed in order to evaluate the parameters that influenced the clinical outcome. Age, sex, aetiology, level and duration of symptoms did not show any 
Table 2 Number of platelets counted in whole blood and in platelet-rich plasma (PRP), platelet concentration factor and number of bone marrow mononucleated cells (BMMNCs) freshly isolated and transplanted in the horses

\begin{tabular}{lllll}
\hline Horse & \multicolumn{2}{l}{ Platelet count $\left(\times 10^{3} / \mu \mathrm{m}^{3}\right)$} & $\begin{array}{l}\text { Platelet concentration } \\
\text { factor }\end{array}$ & $\begin{array}{l}\text { BMMNC count } \\
\left(\text { cells } \times 10^{6}\right)\end{array}$ \\
\cline { 2 - 3 } & Whole blood & PRP & & 1.5 \\
\hline H1 & 150 & 620 & 4.1 & 3.0 \\
H2 & 130 & 680 & 5.2 & 2.5 \\
H3 & 130 & 630 & 4.9 & 0.8 \\
H4 & 120 & 730 & 6.1 & 3.3 \\
H5 & 130 & 540 & 4.2 & 4.0 \\
H6 & 160 & 560 & 3.5 & 1.1 \\
H7 & 120 & 880 & 7.3 & 1.0 \\
H8 & 220 & 960 & 4.4 & 1.0 \\
H9 & 210 & 1040 & 5.0 & 2.1 \\
H10 & 68 & 474 & 7.0 & 0.8 \\
H11 & 160 & 960 & 6.0 & 0.9 \\
H12 & 150 & 920 & 6.1 & 2.4 \\
H13 & 120 & 768 & 6.4 & 1.9 \\
Mean & 144 & 751 & 5.4 & 1.1 \\
Standard deviation (SD) & 40 & 185 & 1.2 & \\
\hline
\end{tabular}

influence on the results. PRP was the only parameter that predicted 'healing time' (Fig. 3). In particular, significantly faster recovery $(p=0.049)$ was observed in cases of PRP with more than $750 \times 10^{3} / \mu$ l platelets $(2.8 \pm 0.4$ months $)$ with

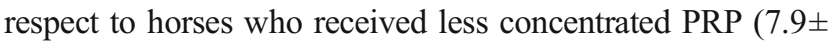
4.3 months) (Fig. 4). In our series no effect of the BMMNCs concentration was found. However, the two horses who did not go back to competition received both the lower amount of platelets and BMMNCs (Fig. 5).

\section{Discussion}

In this study we evaluated the use of combined administration of PRP gel and freshly isolated autologous BMMNCs for the treatment of musculoskeletal overuse injuries in competition horses. As for humans athletes, tendon and ligament overuse injuries are frequent and severe pathologies in competition horses [18]. Tendons heal slowly, and rarely recover their original properties of strength, elasticity and function. Moreover, the increased stiffness of scar tissue also exerts increased strain, resulting in a high incidence of recurrent injury $[18,19]$. The lack of efficacy of current treatment strategies explains the wide research and numerous studies in this animal model. The knowledge gained from animals subjected to mechanically demanding activities may be useful for advances in this research field and could be subsequently translated into the clinical practice in humans.

Regenerative medicine aims to favour the healing of the tissue recovering its original functional properties.

Recently, there has been particular interest in platelets and mesenchymal cell therapy for tendon repair, especially in

Table 3 Quantitative ELISA analysis of TGF $\beta 1$, PDGF-AB, VEGF, IGF, EGF and IL- $1 \beta$ content in plasma and CaCl ${ }_{2}$ activated PRP

\begin{tabular}{|c|c|c|c|c|c|c|c|c|c|c|c|c|}
\hline \multirow[t]{2}{*}{ Horse } & \multicolumn{2}{|c|}{ TGFß1 (pg/ml) } & \multicolumn{2}{|c|}{ PDGF-AB (pg/ml) } & \multicolumn{2}{|c|}{ VEGF (pg/ml) } & \multicolumn{2}{|c|}{ IGF (ng/ml) } & \multicolumn{2}{|c|}{ EGF (pg/ml) } & \multicolumn{2}{|c|}{ IL-1ß (pg/ml) } \\
\hline & Plasma & aPRP & Plasma & aPRP & Plasma & aPRP & Plasma & aPRP & Plasma & aPRP & Plasma & aPRP \\
\hline H5 & 440 & 3660 & 112 & 570 & 12.3 & 162 & 159 & 207 & 4 & 4.4 & 1.1 & 2.1 \\
\hline H6 & 230 & 2450 & 101 & 192 & 9.3 & 154 & 230 & 254 & 3.8 & 5.6 & 1.1 & 2.5 \\
\hline $\mathrm{H} 7$ & 150 & 3200 & 112 & 344 & 10 & 162 & 250 & 395 & 4.4 & 11.2 & 0.9 & 8.3 \\
\hline H8 & 700 & 4080 & 84 & 350 & 11.5 & 203 & 173 & 235 & 1.96 & 4 & 1.4 & 4.6 \\
\hline H9 & 300 & 2470 & 38 & 347 & 10.7 & 154 & 99 & 311 & 1.68 & 3.6 & 1.3 & 2.6 \\
\hline H10 & 920 & 2860 & 58 & 342 & 11.5 & 170 & 242 & 433 & 0.42 & 2.94 & 1.3 & 3.4 \\
\hline H11 & 700 & 2860 & 58 & 353 & 11.5 & 186 & 122 & 237 & 0.94 & 2.94 & 1.6 & 2.8 \\
\hline H12 & 700 & 2860 & 58 & 359 & 18.6 & 162 & 91 & 242 & 1.02 & 2.36 & 2 & 5.4 \\
\hline Mean & 517.5 & 3055.0 & 77.6 & 357.1 & 11.9 & 169.1 & 170.7 & 289.2 & 2.3 & 4.6 & 1.3 & 3.9 \\
\hline SD & 275.9 & 568.2 & 28.5 & 102.2 & 2.9 & 17.1 & 64.2 & 83.0 & 1.6 & 2.8 & 0.3 & 2.1 \\
\hline
\end{tabular}




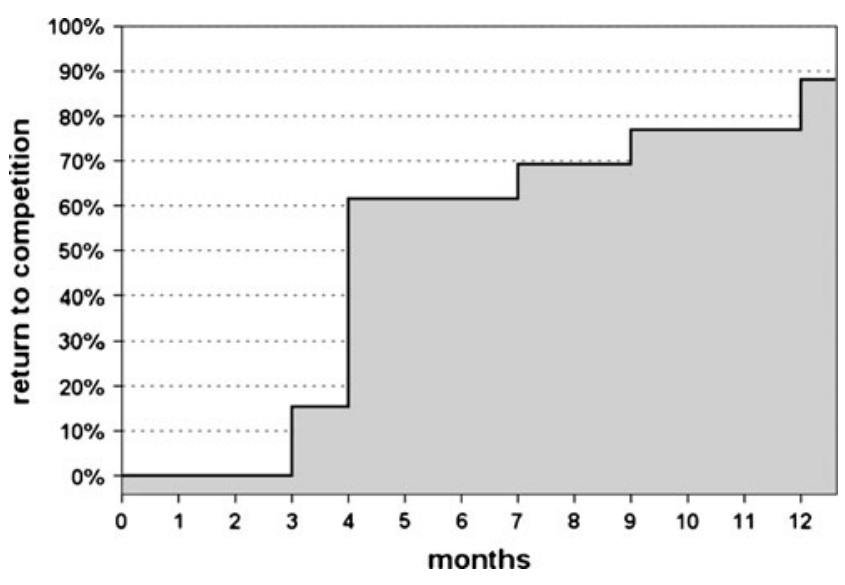

Fig. 1 Return to competition and time required: 11 horses (84.6\%) were able to return to competition, whereas two of them did not go back to races

racehorses. Several studies have tested the efficacy in tendon repair of cultured and expanded BMMNCs [20, 21], whereas there are only two studies investigating the potential of freshly harvested and transplanted BMMNCs in promoting horse tendon healing, with interesting results [10, 22]. In our study, the one-step BMMNC isolation was preferred, avoiding the laboratory culture expansion, that requires a three-week period and high costs. An increasing number of surgeons prefer the injection of marrow concentrate $[10,23]$ because it allows concentration of the relatively few stem cells, and production of a cell pool with minimal cell manipulation and with no risk of cell transformation during in vitro growth. Moreover, reports indicate that laboratory cell handling affects and limits bone marrow proliferative and functional capacities after a prolonged culture, leading to a reduced repair potential [4]. Furthermore PRP is a simple, low cost and minimally-invasive procedure, and is currently being tested in different fields of medicine for its

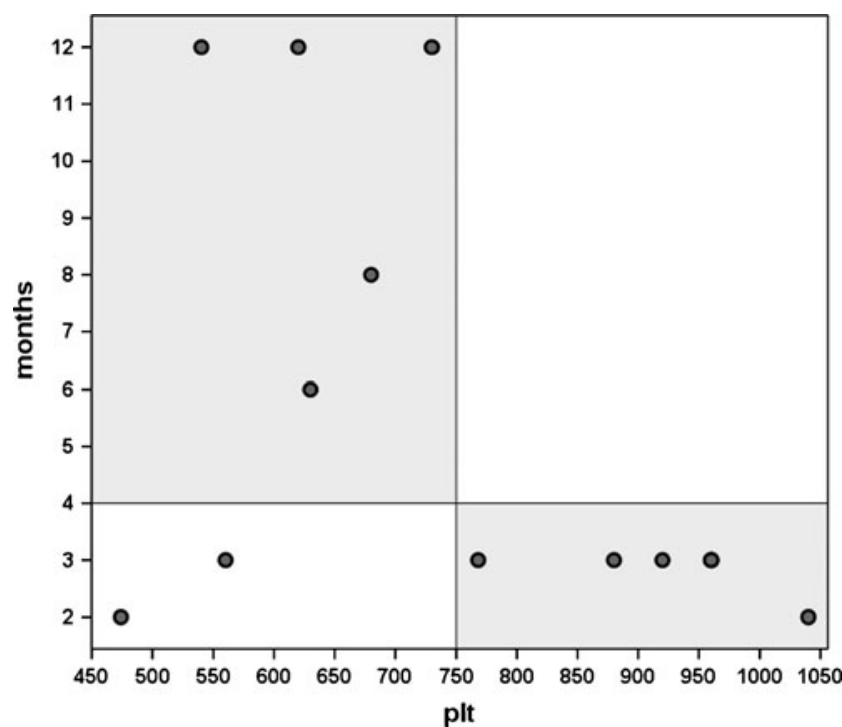

Fig. 3 Correlation between platelet concentration and healing time

ability to aid the regeneration of tissue with a low healing potential [5]. Preclinical and clinical studies support its use for tendon and ligamentous lesions [12, 13]; in fact, PRP has the theoretical advantage of containing various GFs and molecules with a natural balance of anabolic and catabolic functions, capable of optimising the tissue environment in favour of the healing process [24].

Response to injury is coordinated and regulated by mediators and cellular events; in this view, the association of both platelet derived GFs and MSCs would reasonably appear to be a potentially valid and successful treatment approach. Moreover, PRP gel may serve also as a scaffold to homogeneously entrap and retain autologous MSCs in the lesion site, in addition to increasing their proliferation and therefore enhance sinergically the healing capacity.
Fig. 2 Ultrasound evaluation before (a) and after (b) the treatment at one-month followup (horse H6)
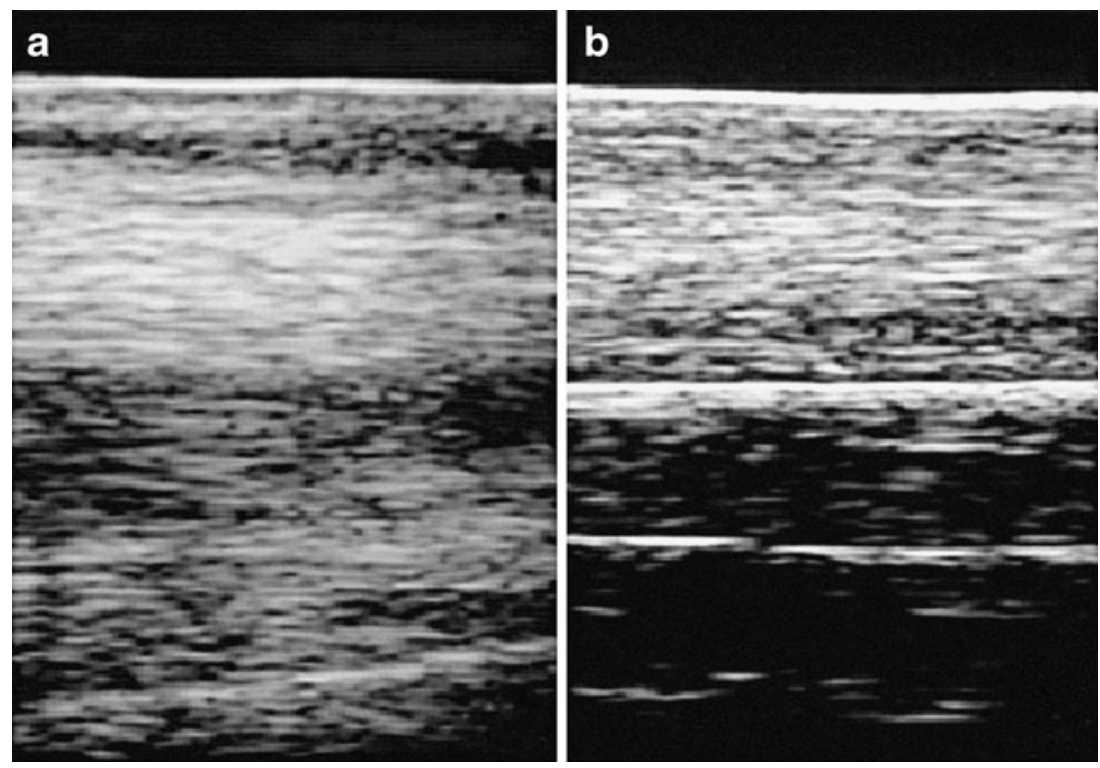


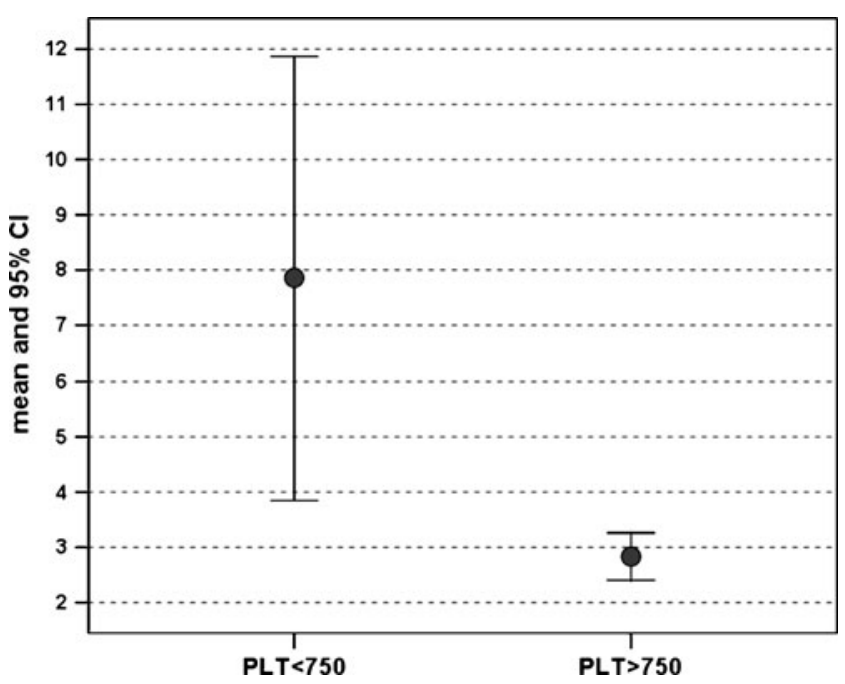

Fig. 4 Mean healing time in relation to the platelet (PLT) number

Although many in vitro or in vivo investigations of the efficacy of PRP or pluripotent cell transplant separately on tendon regeneration [10-13], to our knowledge there is little literature investigating the efficacy of simultaneous administration of both. Del Bue and colleagues showed promising findings evaluating the tendon repair potential of the association between platelet concentrates and allogenic expanded adipose-tissue derived mesenchymal stem cells in 16 horses affected by tendonitis; they found a functional recovery in 14 treated horses [15].

We analysed 13 competition horses affected by overuse musculoskeletal injuries and treated with PRP gel and freshly isolated autologous BMMNCs. Eleven horses
$(84.6 \%)$ were able to return to competition and lameness was solved in all of the cases. We also observed that the final outcome was not influenced by the basal condition, thus demonstrating that recalcitrant and more impairing lesions might also benefit from this treatment. No age and sex influence was documented.

Further analysis was performed to assess factors that may influence the clinical outcome. In particular, the proper dose of GFs and cells to be administrated are of primary importance, and need to be determined in order to achieve better results. GFs are potent molecules, and small variations in their concentrations can produce very different effects. The PRP data are in agreement with those of Marx, who stated that a PRP concentrate should reach approximately $400 \%$ (4x) of the peripheral blood platelet count [25]. We observed that, at the ranges used in this study, a higher number of platelets correlates with a better clinical outcome, whereas no influence was detected analysing the different BMMNCs concentrations. Nevertheless, the two horses that did not go back to competition received both the lower amount of platelets and BMMNCs. However, the authors firmly believe that the number of cells to be transplanted is not the only parameter to be assessed, but also their proliferative and regenerative capacity should be considered particularly when BMMNCs are isolated from aged or sick animals.

We evaluated GFs and IL- $1 \beta$ concentration in plasma and aPRP of eight animals in order to document their increased concentration after PRP activation. No correlation between GFs concentration and clinical outcome was found in this study, probably due to the low number and variety of cases. In fact, important variations in GF concentrations
Fig. 5 Return to races in relation to both platelet (PLT) $\left(\times 10^{3} / \mu \mathrm{l}\right)$ and bone marrow mononucleated cell (BMMNC) $\left(\times 10^{6}\right)$ number

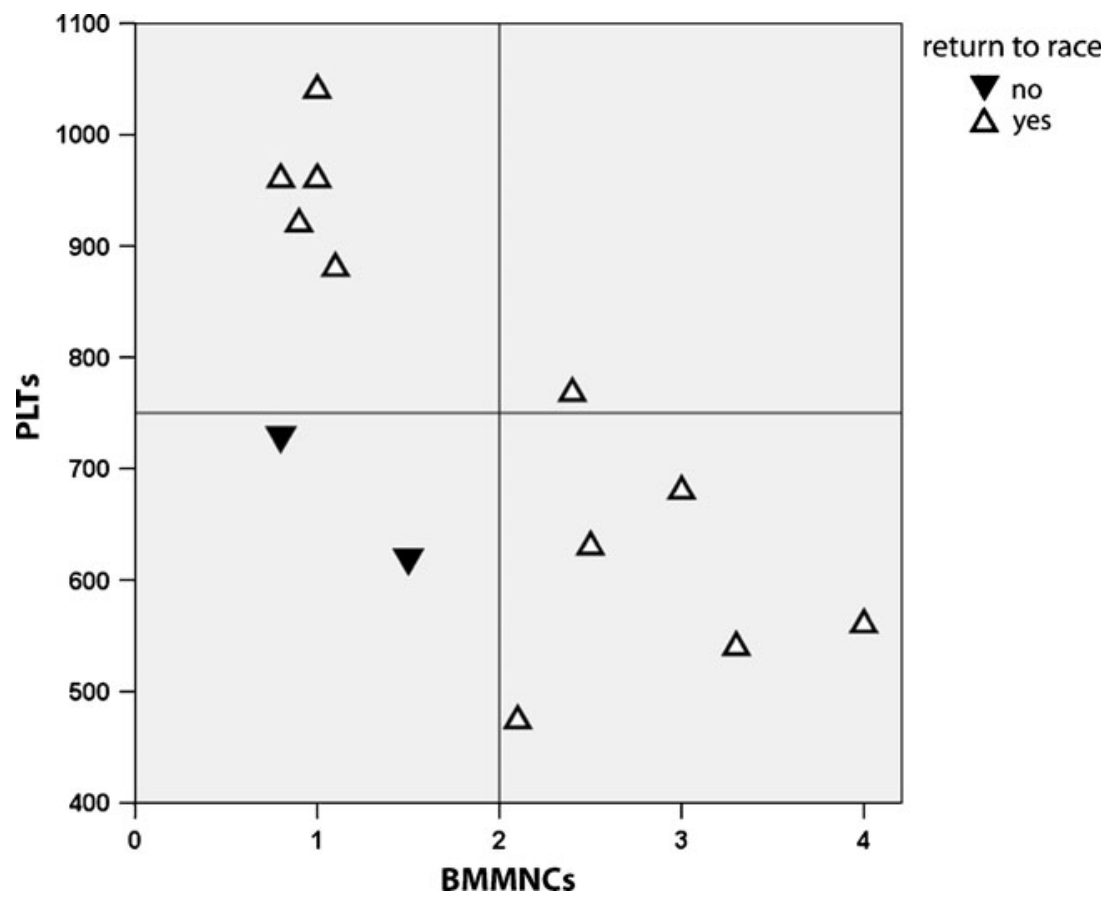


were also detected between subjects having very similar platelet counts, as reported by some authors [26, 27]. Platelets are likewise a source of IL-1ß that is involved to varying degrees in stimulating cell chemotaxis, proliferation and maturation, modulating inflammatory molecules and attracting leukocytes [28]. Whether its effects could exert an effective negative role in tissue healing needs to be determined.

Our study has some limitations. First, we do not know what the individual contribution of PRP and BMMNCs to tendon healing is. Nevertheless, clinical observation suggests that treatment with PRP and BMMNCs might be a promising therapy in the management of these overuse injuries, which have low healing potential with traditional therapies. We evaluated two musculoskeletal injuries, both due to overuse, with the same microtraumatic chronic damage mechanism, and affecting connective tissues with low vascularity, cellularity and healing potential; the groups were homogeneous for all aspects, also for the response to treatment, thus demonstrating their similarity and justifying their collective evaluation. Other weak points of our study are the lack of a control group and of histological evaluation. However, this was not a preclinical trial, it was rather an analysis of the preliminary findings obtained with this new combined biological approach in sport horses. This evaluation lacks the high homogeneity of the animal model in scientifically designed trials, but on the other hand allows observation of the outcome in a specific study population; in fact, competition horses are involved in highly demanding activities, thus being a similar model to the high mechanical overload typical of human athletes. Finally, other than the potential of this combined one step approach for the treatment of refractory lesions that failed previous treatments, with fast return to previous activity level in all the horses including racing in $84.6 \%$ of the cases, we demonstrated that a higher number of platelets influence the recovery speed (which should be considered in future animal and human applications), whereas horses treated with both a low amount of platelets and BMMNCs failed to return to race competition.

Future randomised controlled studies, applied to a greater number of horses, are needed to confirm these results. Moreover, PRP or BMMNCs concentrations effects need to be investigated, as well as the role of the many other bioactive molecules obtained and delivered to the lesion site with this combined biological procedure.

Acknowledgements This work was partially supported by the project "Regione Emilia Romagna Programma di Ricerca Regione-Università 2007-2009” (Regenerative Medicine in Osteoarticular Disease).

Conflict of interest The authors declare that they have no conflict of interest.

\section{References}

1. Lind M, Jakobsen BW, Lund B, Hansen MS, Abdallah O, Christiansen SE (2009) Anatomical reconstruction of the medial collateral ligament and posteromedial corner of the knee in patients with chronic medial collateral ligament instability. Am J Sports Med 37(6):1116-1122

2. Kettunen JA, Kvist M, Alanen E et al (2002) Long term prognosis for jumper's knee in male athletes. A prospective follow-up study. Am J Sport Med 30(5):689-692

3. Cook JL, Kiss ZS, Khan KM et al (2004) Anthropometry, physical performance, and ultrasound patellar tendon abnormality in elite junior basketball players: a cross sectional study. Br J Sports Med 38:206-209

4. Koch TG, Berg LC, Betts DH (2009) Current and future regenerative medicine-principles, concepts, and therapeutic use of stem cell therapy and tissue engineering in equine medicine. Can Vet J 50:155-165

5. Anitua E, Andia I, Ardanza B et al (2004) Autologous platelets as a source of proteins for healing and tissue regeneration. Thromb Haemost 91:4-15

6. Borzini P, Mazzucco L (2007) Platelet-rich plasma (PRP) and platelet derivatives for topical therapy. What is true from the biologic view point? ISBT Sci Ser 2:272-281

7. Huang Q, Wang YD, Wu T et al (2009) Preliminary separation of the growth factors in platelet-rich plasma: effects on the proliferation of human marrow-derived mesenchymal stem cells. Chin Med J (Engl) 122(1):83-87

8. Dallari D, Fini M, Stagni C et al (2006) In vivo study on the healing of bone defects treated with bone marrow stromal cells, platelet-rich plasma, and freeze-dried bone allografts, alone and in combination. J Orthop Res 24(5):877-888

9. Chong AK, Chang J, Go JC (2009) Mesenchymal stem cells and tendon healing. Front Biosci 14:4598-4605

10. Crovace A, Lacitignola L, Rossi G, Francioso E (2010) Histological and immunohistochemical evaluation of autologous cultured bone marrow mesenchymal stem cells and bone marrow mononucleated cells in collagenase-induced tendinitis of equine superficial digital flexor tendon. Vet Med Int 2010:250978

11. Smith RKW, Korda M, Blunn GW et al (2003) Isolation and implantation of autologous equine mesenchymal stem cells from bone marrow into the superficial digital flexor tendon as a potential novel treatment. Equine Vet 35:99-102

12. Sánchez M, Anitua E, Azofra J, Andía I, Padilla S, Mujika I (2007) Comparison of surgically repaired Achilles tendon tears using platelet-rich fibrin matrices. Am J Sports Med 35(2):245-251

13. Filardo G, Kon E, Della Villa S, Vincentelli F, Fornasari PM, Marcacci M (2010) Use of platelet-rich plasma for the treatment of refractory jumper's knee. Int Orthop 34(6):909-915

14. Kon E, Filardo G, Delcogliano M et al (2009) Platelet-rich plasma: new clinical application: a pilot study for treatment of jumper's knee. Injury 40(6):598-603

15. Del Bue M, Riccò S, Ramoni R et al (2008) Equine adipose-tissue derived mesenchymal stem cells and platelet concentrates: their association in vitro and in vivo. Vet Res Commun 32(S1):S51S55

16. Schnabel LV, Lynch ME, Van der Meulen MCH et al (2009) Mesenchymal stem cells and insulin-like growth factor-I geneenhanced mesenchymal stem cells improve structural aspects of healing in equine flexor digitorum superficialis tendons. J Orthop Res 27(10):1392-1398

17. McCarrel T, Fortier L (2009) Temporal growth factor release from platelet-rich plasma, trehalose lyophilized platelets, and bone marrow aspirate and their effect on tendon and ligament gene expression. J Orthop Res 27(8):1033-1042 
18. Dowling BA, Dart AJ, Hodgson DR et al (2000) Superficial digital flexor tendonitis in the horse. Equine Vet J 25:913-925

19. Fortier LA, Smith RKW (2008) Regenerative medicine for tendinous and ligamentous injuries of sport horses. Vet Clin Equine 24:191-201

20. Guest DJ, Smith MR, Allen WR (2008) Monitoring the fate of autologous and allogenic mesenchymal progenitor cells injected into the superficial digital flexor tendon of horses. Preliminary study. Equine Vet J 40:178-181

21. Arthur A, Zannettino A, Gronthos S et al (2009) The therapeutic applications of multipotential mesenchymal/stromal stem cells in skeletal tissue repair. J Cell Physiol 218:237-245

22. Lacitignola L, Crovace A, Rossi G, Francioso E (2008) Cell therapy for tendinitis, experimental and clinical report. Vet Res Commun 32(Suppl 1):S33-S38

23. Giannini S, Buda R, Vannini F et al (2009) One-step bone marrowderived cell transplantation in talar osteochondral lesions. Clin Orthop Relat Res 467(12):3307-3320
24. Anitua E, Sanchez M, Nurden AT et al (2006) New insights into and novel applications for platelet-rich fibrin therapies. Trends Biotechnol 24:227-234

25. Marx RE (2000) Quantification of growth factor levels using a simplified method of platelet-rich plasma gel preparation. J Oral Maxillofac Surg 58:300-301

26. Borzini P, Mazzucco L (2005) Tissue regeneration and in-loco administration of platelet derivatives. Clinical outcome, heterogeneous products, eterogeneity of the effector mechanisms. Transfusion 35:1759-1767

27. Weibrich G, Kleis WKG, Hitzler WE, Hafner G (2005) Comparison of the platelet concentrate collection system with the plasma-rich-in-growth-factors kit to produce platelet-rich plasma: a technical report. Int J Oral Maxillofac Implants 20:118123

28. Lindemann S, Tolley ND, Dixon DA et al (2001) Activated platelets mediate inflammatory signaling by regulated interleukin $1 \beta$ synthesis. J Cell Biol 154(3):485-490 